\title{
Digital phenotyping for mental health of college students: a clinical review
}

\author{
Jennifer Melcher, Ryan Hays, John Torous
}

Division of Digital Psychiatry, Beth Israel Deaconess Medical Center, Harvard Medical School, Boston, Massachusetts, USA

\section{Correspondence to} Dr John Torous, Psychiatry, Beth Israel Deaconess Medical Center, Boston, Massachusetts, USA; jtorous@bidmc.harvard. edu

Received 4 August 2020 Revised 2 September 2020 Accepted 3 September 2020 Published Online First 30 September 2020

\section{Check for updates}

(c) Author(s) (or their employer(s)) 2020. No commercial re-use. See rights and permissions. Published by BMJ.

To cite: Melcher J, Hays R, Torous J. Evid Based Ment Health 2020:23:161-166.

\section{ABSTRACT}

Experiencing continued growth in demand for mental health services among students, colleges are seeking digital solutions to increase access to care as classes shift to remote virtual learning during the COVID-19 pandemic. Using smartphones to capture real-time symptoms and behaviours related to mental illnesses, digital phenotyping offers a practical tool to help colleges remotely monitor and assess mental health and provide more customised and responsive care. This narrative review of 25 digital phenotyping studies with college students explored how this method has been deployed, studied and has impacted mental health outcomes. We found the average duration of studies to be 42 days and the average enrolled to be 81 participants. The most common sensor-based streams collected included location, accelerometer and social information and these were used to inform behaviours such as sleep, exercise and social interactions. $52 \%$ of the studies included also collected smartphone survey in some form and these were used to assess mood, anxiety and stress among many other outcomes. The collective focus on data that construct features related to sleep, activity and social interactions indicate that this field is already appropriately attentive to the primary drivers of mental health problems among college students. While the heterogeneity of the methods of these studies presents no reliable target for mobile devices to offer automated help-the feasibility across studies suggests the potential to use these data today towards personalising care. As more unified digital phenotyping research evolves and scales to larger sample sizes, student mental health centres may consider integrating these data into their clinical practice for college students.

\section{INTRODUCTION}

Against the backdrop of COVID-19, college mental health services, like the rest of the field, are moving online. While synchronous telehealth solutions like telepsychiatry offer continuity of care, the need for asynchronous solutions that can increase access to care such as digital phenotyping is now patent. The astounding number of college students experiencing mental health problems is not new to this period of COVID-19, with the 2019 American College Health Association National College Health Assessment revealing that 13\% of college students seriously considered suicide within the past year. ${ }^{1}$ Reasons for this increase in students with diagnosed mental health conditions, from $22 \%$ to $36 \%$ from 2007 to 2017 , remain unclear but include a decrease in stigma leading to more help seeking and increases in stress faced by students among other contributing factors. ${ }^{2}$ Now, a national survey of college students in April of 2020 suggests that the prevalence of anxiety, depression and social isolation has only increased during the coronavirus pandemic with $20 \%$ of students reporting that their mental health has significantly worsened under COVID $-19 .^{3}$

These early survey results show a growing mental health crisis in college students and the urgent need for mental health solutions adapted to an increasingly remote lifestyle and 'social distancing'. After being sent home from colleges and their campus mental health centres, $55 \%$ of students reported they did not know where to go for mental health help. ${ }^{3}$ As colleges seek to understand how students are coping, provide personalised care and offer preventive mental health services-they will need to use digital phenotyping. Defined as using data from sensors and interactions on personal digital devices to measure behaviour, digital phenotyping is actually not a novel concept and has already been used to offer new insights into college mental health. ${ }^{4-6}$

College students are particularly well suited to digital phenotyping as smartphone ownership among college-aged adults is higher than any other age group. ${ }^{7}$ Students' response rates to active data prompts in previous digital phenotyping research shows that students are accepting of and adherent to digital phenotyping apps on their mobile devices. ${ }^{8}$ Additionally, a study of active data responses among college students during the COVID-19 pandemic demonstrates that students can adopt these technologies with ease and speed, allowing immediate data collection and monitoring during rapidly changing circumstances. This pilot studies demonstrated that digital devices are already being used to collect data on college student mental health over time in a way that single time point surveys cannot. ${ }^{9}$ Further implementation of digital phenotyping in colleges can identify the reasons that students may be experiencing mental health problems as well as offer timely treatments. Data from smartphones paired with clinical assessment data can reveal what behaviours or combination of behaviours are correlated with mental health problems, getting at the root of why colleges are seeing this number of mental health problems. Then, personalised psychiatric interventions can be delivered to students' smartphones when smartphone data reveals that the student is struggling.

The potential of digital phenotyping is best understood in terms of the data behind it.

Digital phenotyping generally involves constant mobile sensing and collection of data through 
smartphones or other technology such as wearable smartwatches. One of the greatest benefits to leveraging smartphones in digital phenotyping studies is their ability to collect many disparate variables concurrently. In general, data types are categorised as active or passive data. Active data are that in which patient experience is captured through conscious user engagement such as a student reporting thoughts of suicide; passive data are that in which patient experience is captured without conscious user engagement (ie, the user does not submit experiential data explicitly) such as the phone recording that a student has not slept in the past 36 hours.

While the ability of devices to collect this wide array of data offer many potential benefits such as offering personalised psychiatric care, encouraging preventive care and providing the necessary context for self-help based just-in-time adaptive interventions-the actual use of digital phenotyping in college students and resulting insights from that data remain unclear. In this review, we aim to summarise the current practices in digital phenotyping for college student mental health in order to describe how digital phenotyping can best serve college students and to identify best practices.

\section{METHODS}

Articles were found from a search of Google Scholar records (up to May 2020) with keywords including 'college students' or 'college mental health' and 'digital phenotyping', or 'mobile sensing', or 'passive sensing'. Articles were screened to ensure that the population of interest was college students. Articles which proposed studies or study methodologies but did not include completion of a study or offer data were not included. Studies that collected active data but no passive data were not included, but those that collected only passive data were still considered digital phenotyping and included in this analysis. Because digital phenotyping studies vary widely, a systematic review was not feasible. Instead, the 25 articles included present a narrative review of the current practices of digital phenotyping studies for college student mental health.

\section{PRESENTATION}

\section{Devices: Smartphones}

The ubiquity of smartphones allows for mobile sensing to be applied on a large scale, particularly among the college population wherein it is estimated that $96 \%$ of US adults ages $18-29$ years own and operate smartphones regularly. ${ }^{7}$ Despite this, inconsistencies persist in how data are collected via smartphones among digital phenotyping research for college students. Some studies made an effort to provide students with a study phone in order to standardise the quantity and quality of data being collected. However, students were less likely to carry a secondary phone or switch to a new phone, leading to a disparity in data quality among the group that used study phones as their secondary device. ${ }^{81011}$ Other studies distributed study phones to all participants, ${ }^{12} 13$ or restricted participation to those individuals with android phones, excluding any iPhone operating systems (IOS). ${ }^{14-22}$ This is because IOS systems prohibit apps from running constantly in the background and the app approval process to the Apple App Store is more challenging.

\section{Devices: Wearables}

One fundamental complication of using a mobile device to collect passive data on an individual's movement is that it assumes that an individual will always have their mobile device on their person as they move. Because this is not always the case, wearable technology such as smartwatches and activity trackers have also been employed by digital phenotyping studies to capture passive data and construct features primarily related to activity and sleep. ${ }^{15} 1823-25$ Data from wearables are thought to be more accurate or capture more data, allowing more nuanced feature constructions such as sleep efficiency and when sleep was restless. ${ }^{25}$ Wearables also offer physiological data features such as heart rate and electrodermal activity. One study sought to determine whether a wearable could detect heart rate enough to make inferences about an individual's depression, but they did not find any significant correlation. ${ }^{23}$ Studies using wearables for physiological data streams found that reliable data were sparse as external factors such as temperature, movement and humidity interfered with accurate readings. ${ }^{1923}$ Additionally, wearables had a short battery life. Some had to be charged every night, running the risk that they would be forgotten or worn incorrectly thus leaving gaps or inconsistencies in data. ${ }^{23} 25$

\section{$\mathrm{N}$ and length of study}

Digital phenotyping research on college students seems to have little to no consensus on the scope of each study in terms of the duration of the study and the number of participants. Study length varied from as short as 1 week up to three college semesters with an average of 42 days (excluding one study of unclear duration). ${ }^{1226}$ Because these studies focus specifically on college students, research is often constrained to the length of college semesters. Many researchers have adopted a duration of 10 weeks as the standard duration, although there is no research comparing how length of digital phenotyping studies impact results. ${ }^{8} 27-29$ Likewise, the number of college students included in these studies varies widely from as few as 15 to as many as 350 with an average of 81 participants (see table 1 ). ${ }^{22}{ }^{24}$ With no consensus around participants or duration of study, the field of digital phenotyping for college students lacks clear guidance on best practices.

\section{Data set}

Further, the field of college digital phenotyping is defined by a few key studies with publicly accessible data sets. Researchers are making use of these existing data to run new analyses and report new findings. ${ }^{10121827}$ One of the more robust data sets was gathered in 2011 and is still being analysed. ${ }^{12}$ The repeated use old data runs the risk of using increasingly outdated and now irrelevant information in a quickly evolving field to make predictions and inferences of students today.

\section{Active data}

As mentioned, digital phenotyping often involves the collection of both active and passive data. However, while passive data streams fall into a few discrete categories, methods of collecting active data cover a wide range. Thirteen, or $52 \%$ of studies in this analysis included some active data collection. Generally, active data collection involves ecological momentary assessments (EMAs) distributed to a participant through their mobile device. These often appear in the form of short surveys derived from gold-standard clinical assessments or questions related to the focus of the research, but other types of EMAs such as journals or mobile photographic metres are also used. ${ }^{814-182223262930}$ The administration of EMAs to participants' devices varies from as infrequently as once a week to as often as eight times a day with little justification as to why these methods were used. ${ }^{82327}$ The variety of types of EMAs distributed, the specificity of survey EMA questions, and the differences in frequency of EMAs 


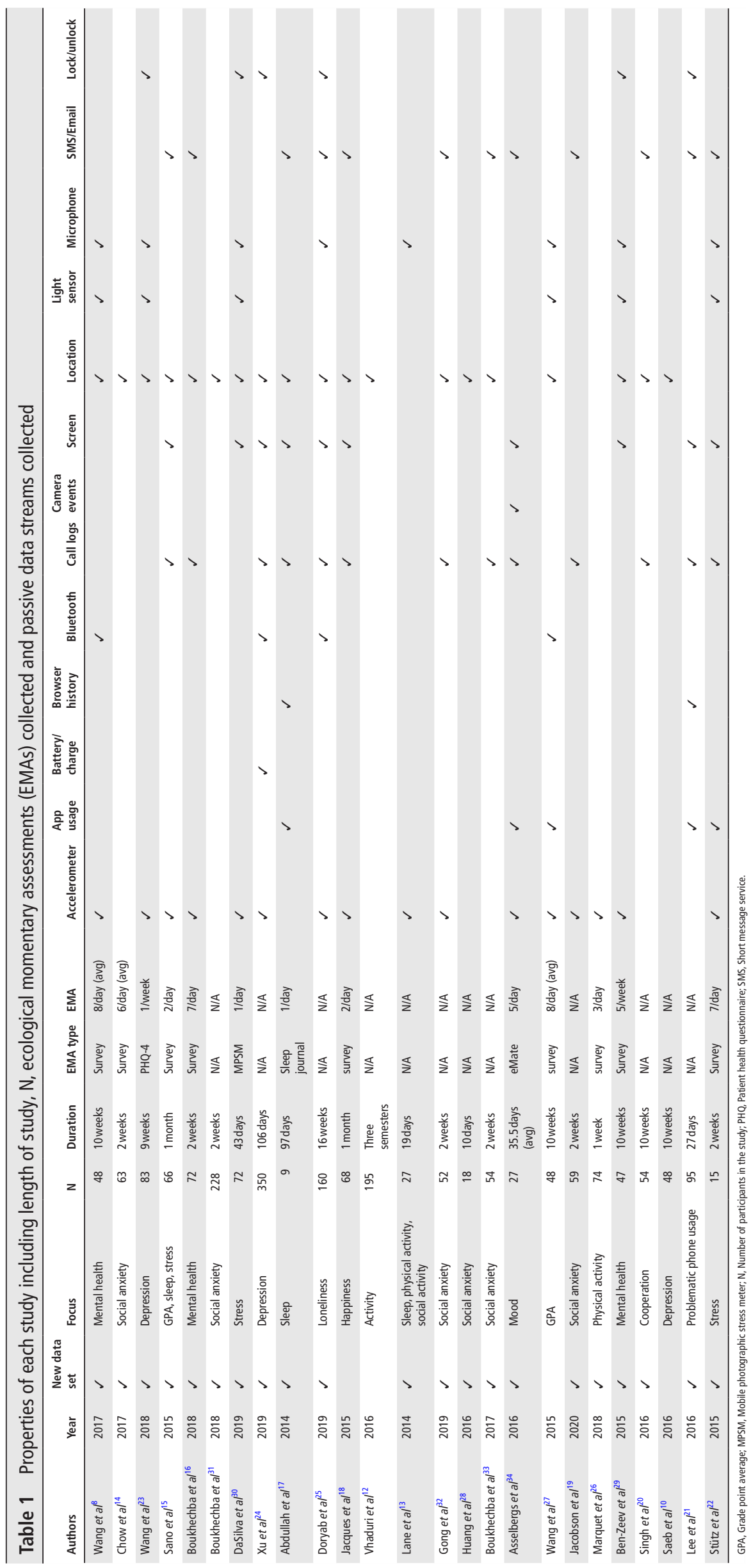




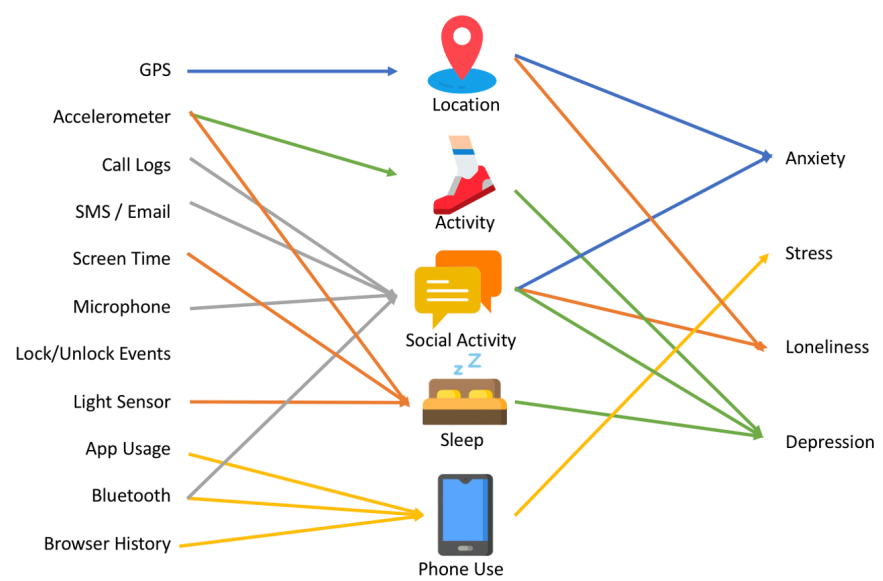

Figure 1 Feature constructions from passive data streams.

provides little guidance for how these studies should be carried out going forward and limits the generalisability of findings.

\section{Passive data}

Smartphones lend the unique opportunity to collect a variety of passive data streams from a user which can then be used to construct features (ie, knowing when someone's phone was still for a long time and there was no light can give insight to a participant's sleep patterns) (see (figure 1).

Identifying which of these data streams or combination of data streams are predictors or signals of mental health problems is often a challenge in digital phenotyping studies. The most popular data streams have generally been location, accelerometer and social information (see figure 2). Here we examine which data streams are employed for different feature constructions and which data streams are most widely accepted and most useful in analysis.

\section{Location}

Location data were most commonly collected through a mobile device's global positioning system (GPS), returning a stream of geographical coordinates. Seventy-six per cent of studies in this analysis collected location data on participants. 89 12 14-18 20 23-25 28-33 One common analysis of location data includes location mapping or classifying specific location coordinates as important locales such as a participant's home, classrooms and study spaces. $81012141618232527-33$

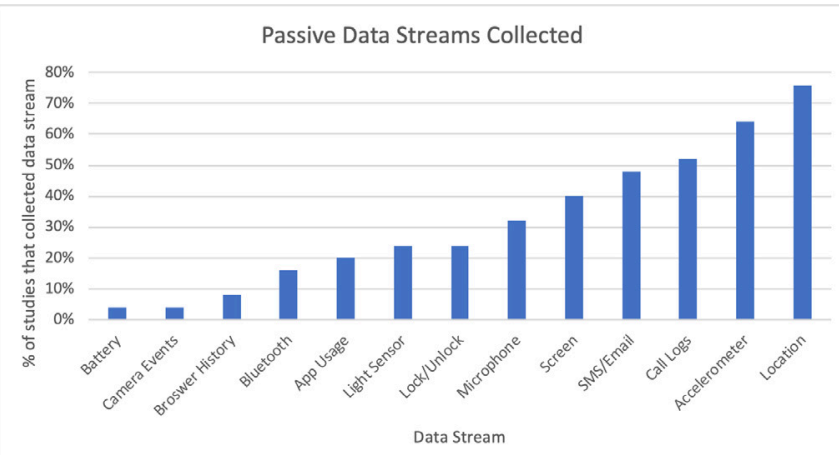

Figure 2 Most popular passive data streams collected.

\section{Accelerometer}

A smartphone's accelerometer records the movement of the phone and can be used to understand activity levels of a participant and was collected by $64 \%$ of studies in this analysis. ${ }^{89131516181922-2629303234}$ Because reduced levels of activity can be indicators of stress, depression or anxiety, previous studies have correlated scores on clinical measurements in these areas with accelerometer data to show that clinical outcomes can be inferred from passive data sensing. ${ }^{8} 1216232932$ Accelerometer data can also be used in conjunction with other features to calculate an individual's sleep habits (ie, the phone was stationary). ${ }^{813152325272930}$ One limitation of accelerometer data from smartphones is that not everyone carries their phone with them constantly, so the phone may not capture the true level of activity of a participant.

\section{Social Information}

Given that changes in social behaviour are often both symptoms and signs of many mental illnesses, interest in using smartphones to gain insights into these domains has been high. Fifty-two per cent of the studies in this analysis collected participants' call logs, and 48\% collected participants' SMS (texting) or email activity to analyse the frequency and duration of calls, emails and texts. ${ }^{15-22242532-34}$ Some studies have looked at communication data in conjunction with location data to understand social behaviours and infer outcomes such as social anxiety, depression, loneliness and even grade point average (GPA). ${ }^{1516181924253233}$ Most studies anonymised social data so that phone numbers could not be found; however, some had participants submit the phone numbers of their closest contacts to monitor those social dynamics specifically. ${ }^{25}$ Social data streams can reveal much about an individual's social behaviour, but individuals are far less willing to share these data given privacy concerns. ${ }^{35}$ Additionally, smartphone operating systems are also recognising privacy risks, with access to these social features among the first to be revoked by Apple and Google. It is important to note that these studies collected solely SMS messaging data and phone calls through cell providers. This ignores any social communication through instant messaging apps (ie, iMessage, WhatsApp, Facebook Messenger) or internet calling apps (ie, FaceTime, WhatsApp, Skype). Internet-based apps have become popular in recent years and their omission in previous digital phenotyping research challenges the validity of these measures. However, due to valid privacy concerns-it is not feasible to collect such data in real time in a digital phenotyping manner.

\section{Screen time}

Correlations have been drawn between screen time and different mental health problems, especially among college-age people, ${ }^{36}$ so gathering screen time data in digital phenotyping studies is particularly relevant and was used by $40 \%$ of the studies in this analysis. ${ }^{15} 171821222425293034$ In some cases, screen time was used to predict loneliness or happiness. ${ }^{18} 25$ However, more commonly, screen time was used to construct sleep estimates (sleep onset, duration and wake time) as the screen was likely off for the duration of the sleeping period. ${ }^{1729} 30$

\section{Screen lock and unlock events}

Much like screen time, phone lock and unlock events can also be telling of an individual's phone use habits. Generally, these data are gathered to calculate the number of phone checks per day and the duration of phone sessions throughout the day in order 
to draw correlations between phone use behaviour and mental health. ${ }^{21} 23-252930$

\section{Bluetooth and microphone}

Some digital phenotyping studies have also gathered Bluetooth and microphone data from smartphones in an attempt to capture duration and frequency of social interactions. ${ }^{8242527}$ Bluetooth data show what other devices with Bluetooth active are nearby. Assuming that another device with Bluetooth was a proxy for a person, researchers can infer levels of social activity or instances of co-location that an individual experienced. Inferring social activity from Bluetooth data is dependent on the participant as well as their acquaintances keeping their Bluetooth active throughout the duration of the study which runs the risk that social interactions may go unrecorded. Like Bluetooth, a phone's microphone can gather social behaviour by picking up on ambient noise which can be then be categorised as either conversation or voice and other ambient noises. ${ }^{813222325272930}$ Understanding the quantity and frequency of social interactions can inform clinical predictions around social anxiety and isolation, depression, and stress.

\section{Light sensor}

Likewise, smartphones' light sensor was also incorporated into the feature construction for sleep. ${ }^{8} 2223272930$ Assuming that most participants would sleep in the dark, the light sensor can help narrow down the sleeping time frame for a participant as well as their sleep onset and waking times.

\section{App usage and browser history}

Some digital phenotyping studies of college students collected data on what apps and websites participants accessed on their smartphone. ${ }^{17212234}$ Apps and websites that students used were recorded and categorised based on their primary function (ie, entertainment, social media, finance, etc) in order to draw correlations between scores on clinical assessments and phone use behaviour. $^{212234}$

\section{DISCUSSION}

This review highlights the collection of digital phenotyping studies concerning college mental health in order to inform the immediately pressing question of what role this technology should have in care today in the COVID-19 era. Overall, the direction of the field of digital phenotyping studies is promising. The focus on smartphone data streams reveals information about sleep, activity and social interactions, highlighting the collective potential to use these data to offer more personalised and responsive mental health for college students.

However, the heterogeneity in the methods of studies reviewed underscores the lack of standardised best practices and nascency of this work. Most studies could be considered pilot studies with an average of 81 participants per study and a duration of 42 days. The most popular patterns of passive data sensors highlight the most promise for data streams including accelerometer data, location data and screen use data. But because these studies have generally focused on one or two specific mental health conditions (eg, only anxiety for example) among college students, there has not yet been a full investigation of how all of the data streams may be combined to gain a more holistic view of an individual's mental health.

In conjunction with the heterogeneity of methods reviewed, it is important to note that data reporting was also inconsistent among studies reviewed. This limits the ability to compare these studies as well as omits important information. For example, most studies in this analysis did not include information about adherence to the active data collection. Those that did report adherence used a variety of measures. The lack of standardisation of reporting among remotely collected data is not a novel problem. Guidelines exist for reporting these data and should be considered for future digital phenotyping studies. ${ }^{37}$

The results of this review demonstrate that it is both feasible and acceptable to use digital phenotyping methods around college mental health. This suggests that further pilot studies may contribute less to the field as the new and next challenge lies in drawing mental health inferences from these data as well as using this information to improve clinical outcomes for students. The inherent value in adding information such as sleep, social, screen time, activity and location data into clinical formulations and therapeutic plans is clear and in line with the biopsychosocial model of mental health, but the complexity of such work as alluded to remains. Thus, productive next steps include either (1) A deeper phenotyping approach to attempt to glean insights or (2) A broader phenotyping approach to find trends from larger samples of students.

Such a deeper phenotyping approach could, for example, capture new data streams like social media but will quickly raise mounting ethical questions. ${ }^{3}$ For example, one study assessing college students' willingness to share key stroke data revealed high levels of unacceptability. ${ }^{35}$ The second approach is to handle complexity through a larger sample size, via a more unified collaborative effort among colleges consisting of independent studies with standardised procedures, digital phenotyping methods and data reporting. Working together, this consortium of institutions could provide a more comprehensive understanding of mental health among college students and provide a sufficiently large and diverse sample to elucidate behavioural signals from these data.

The ultimate goal of digital phenotyping with college students is not only to understand why students experience these mental health problems, but also offer more personalised and responsive care-be that via an app, online programme or face-toface care. Based on the current evidence, digital phenotyping can directly benefit student mental health today but not yet in a fully scalable manner. As there were no clear trends in our results that offered a reliable target just-in-time adaptive interventions (feedback loops utilzing digital phenotyping data to trigger in-app interventions) - any smartphone or computer programme offering fully automated care based on such data is likely not optimised or accurate. Further sample sizes necessary for reinforcement learning that could match individuals' digital phenotypes to responsive app interventions would require thousands of students-something not seen in the current literature.

As COVID-19 continues to transform mental healthcare and service delivery, colleges are well poised to benefit from digital phenotyping. While these new data cannot yet automate care, future research with standardised practices, reporting and larger sample sizes are the next steps towards using digital phenotyping to inform clinical treatment with college students.

Twitter John Torous @JohnTorousMD

Acknowledgements The authors thank Jeremy Wertheimer for a generous philanthropic gift and support that enables them to pursue this research.

Contributors All authors contributed equally.

Funding The authors have not declared a specific grant for this research from any funding agency in the public, commercial or not-for-profit sectors.

Competing interests JT receives unrelated research support from Otsuka. 
Patient consent for publication Not required.

Provenance and peer review Not commissioned; externally peer reviewed.

Data availability statement No data are available.

ORCID iD

John Torous http://orcid.org/0000-0002-5362-7937

\section{REFERENCES}

1 NCHA-II spring 2019 US reference group executive summary. Available: https:// www.acha.org/documents/ncha/NCHA-II_SPRING_2019_US_REFERENCE_GROUP_ EXECUTIVE_SUMMARY.pdf [Accessed 17 Jul 2020].

2 The healthy minds study 2018-2019 data report. Available: https:// healthymindsnetwork.org/wp-content/uploads/2019/09/HMS_national-2018-19.pdf [Accessed $17 \mathrm{Jul} 2020]$.

3 The impact of COVID-19 on student mental health. active minds, 2020. Available: https://www.activeminds.org/studentsurvey/ [Accessed 17 Jul 2020].

4 Insel TR. Digital phenotyping: technology for a new science of behavior. JAMA 2017;318:1215-6

5 Torous J, Kiang MV, Lorme J, et al. New tools for new research in psychiatry: a scalable and customizable platform to empower data driven smartphone research. JMIR Ment Health 2016:3:e16.

6 Jain SH, Powers BW, Hawkins JB, et al. The digital phenotype. Nat Biotechnol 2015:33:462-3.

7 Pew Research Center: Internet, Science \& Tech. Demographics of mobile device ownership and adoption in the United States. Available: https://www.pewresearch. org/internet/fact-sheet/mobile/ [Accessed 17 Jul 2020].

8 Wang R, Chen F, Chen Z, et al. StudentLife: Using smartphones to assess mental health and academic performance of college students. Mobile Health - Sensors, Analytic Methods, and Applications, 2017. Available: https://home.cs.dartmouth. edu/ xia/papers/mobilehealth17.pdf

9 Taquet M, Quoidbach J, Fried El, et al. Mood homeostasis before and during the coronavirus disease 2019 (COVID-19) lockdown among students in the Netherlands. JAMA Psychiatry 2020. doi:10.1001/jamapsychiatry.2020.2389. [Epub ahead of print: 29 Jul 2020].

10 Saeb S, Lattie EG, Schueller SM, et al. The relationship between mobile phone location sensor data and depressive symptom severity. PeerJ 2016;4:e2537.

11 Striegel A, Liu S, Meng L, et al. Lessons learned from the netsense smartphone study. SIGCOMM Comput Commun Rev 2013;43:51-6.

12 Vhaduri S, Munch A, Poellabauer C. Assessing health trends of college students using smartphones. IEEE Healthcare Innovation Point-Of-Care Technologies Conference, 2016:70-3.

13 Lane ND, Lin M, Mohammod M, et al. BeWell: sensing sleep, physical activities and social interactions to promote wellbeing. Mobile Networks and Applications $2014 \cdot 19 \cdot 345-59$

14 Chow PI, Fua K, Huang Y, et al. Using mobile sensing to test clinical models of depression, social anxiety, state affect, and social isolation among college students. $J$ Med Internet Res 2017;19:e62.

15 Sano A, Phillips AJ, Yu AZ, et al. Recognizing academic performance, sleep quality, stress level, and mental health using personality traits, wearable sensors and mobile phones. Int Conf Wearable Implant Body Sens Netw 2015:2015. doi:10.1109/ BSN.2015.7299420

16 Boukhechba M, Daros AR, Fua K, et al. DemonicSalmon: monitoring mental health and social interactions of college students using smartphones. Smart Health 2018;910:192-203.

17 Abdullah S, Matthews M, Murnane EL, et al. Towards circadian computing: "Early to bed and early to rise" makes some of us unhealthy and sleep deprived. Proceedings of the 2014 ACM International Joint Conference on Pervasive and Ubiquitous Computing, 2014:673.
18 Jaques N, Taylor S, Azaria A, et al. Predicting students' Happiness from physiology, phone, mobility, and behavioral data. Int Conf Affect Comput Intell Interact Workshops 2015;2015:222-8.

19 Jacobson NC, Summers B, Wilhelm S. Digital biomarkers of social anxiety severity: digital phenotyping using passive smartphone sensors. J Med Internet Res 2020;22:e16875.

20 Singh VK, Agarwal RR. Cooperative phoneotypes: exploring phone-based behavioral markers of cooperation. Proceedings of the 2016 ACM International Joint Conference on Pervasive and Ubiquitous Computing, 2016:646-57.

21 Lee $\mathrm{U}$, Lee J, Ko M, et al. Hooked on smartphones: an exploratory study on smartphone overuse among college students. Proc SIGCHI Conf Hum Factor Comput Syst 2014.

22 Stütz T, Kowar T, Kager M, et al. Smartphone based stress prediction, 2015: 240-51.

23 Wang R, Wang W, daSilva A, et al. Tracking depression dynamics in college students using mobile phone and wearable sensing. Proc ACM Interact Mob Wearable Ubiquitous Technol 2018;2:1-26

24 Xu X, Chikersal P, Doryab A, et al. Leveraging routine behavior and contextuallyfiltered features for depression detection among college students. Proc ACM Interact Mob Wearable Ubiquitous Technol 2019:3:1-33. 116:33.

25 Doryab A, Villalba DK, Chikersal P, et al. Identifying behavioral phenotypes of loneliness and social isolation with passive sensing: statistical analysis, data mining and machine learning of smartphone and fitbit data. JMIR Mhealth Uhealth 2019;7:e13209.

26 Marquet O, Alberico C, Hipp AJ. Pokémon go and physical activity among college students. A study using ecological Momentary assessment. Comput Human Behav 2018:81:215-22.

27 Wang R, Harari G, Hao P, et al. SmartGPA: how smartphones can assess and predict academic performance of college students. Proceedings of the 2015 ACM International Joint Conference on Pervasive and Ubiquitous Computing, 2015:295-306

28 Huang $\mathrm{Y}$, Xiong $\mathrm{H}$, Leach $\mathrm{K}$, et al. Assessing social anxiety using GPs trajectories and point-of-interest data. Proceedings of the 2016 ACM International Joint Conference on Pervasive and Ubiquitous Computing, 2016.

29 Ben-Zeev D, Scherer EA, Wang R, et al. Next-Generation psychiatric assessment: using smartphone sensors to monitor behavior and mental health. Psychiatr Rehabil J 2015;38:218-26

30 DaSilva AW, Huckins JF, Wang R, et al. Correlates of stress in the College environment uncovered by the application of penalized generalized estimating equations to mobile sensing data. JMIR Mhealth Uhealth 2019;7:e12084.

31 Boukhechba M, Chow P, Fua K, et al. Predicting social anxiety from global positioning system traces of college students: feasibility study. JMIR Ment Health 2018:5:e10101.

32 Gong J, Huang Y, Chow PI, et al. Understanding behavioral dynamics of social anxiety among college students through smartphone sensors. Information Fusion 2019;49:57-68.

33 Boukhechba M, Huang Y, Chow P, et al. Monitoring social anxiety from mobility and communication patterns. Proceedings of the 2017 ACM International Joint Conference on Pervasive and Ubiquitous Computing, 2017:749-53.

34 Asselbergs J, Ruwaard J, Ejdys M, et al. Mobile phone-based unobtrusive ecological momentary assessment of day-to-day mood: an explorative study. J Med Internet Res 2016;18:e72.

35 Rooksby J, Morrison A, Murray-Rust D. Student perspectives on digital phenotyping: the acceptability of using smartphone data to assess mental health. Proceedings of the Proc SIGCHI Conf Hum Factor Comput Syst, 2019.

36 Wu X, Tao S, Zhang Y, et al. Low physical activity and high screen time can increase the risks of mental health problems and poor sleep quality among Chinese college students. PloS One 2015:10:e0119607.

37 Faurholt-Jepsen M, Geddes JR, Goodwin GM, et al. Reporting guidelines on remotely collected electronic mood data in mood disorder (eMOOD) — recommendations. Trans/ Psychiatry 2019;9:1-10. 